\section{Positive feedback between participation and MPA performance in the Philippines}

Surveys of resource users in villages near MPAs in the Philippines suggests that participation in an MPA planning and management process leads to perceptions of better MPA performance. Participation and perceptions of MPA performance may be part of a positive feedback loop with increased scientific knowledge, perceived personal benefits from the MPA, and self-identification with the natural world.

The authors used surveys of residents from 15 villages in the Philippines, totaling 214 individuals, to examine what factors led to participation in a marine protected area (MPA) planning and ongoing management process. Participation was divided into two categories: active (i.e. participating in monitoring and enforcement activities, attending trainings, etc.) and consultative (i.e. if the user felt themself or their community were consulted in the planning process). Of those users who knew there was an MPA near their village, $74 \%$ were involved in at least one participatory activity, with $21 \%$ involved in monitoring efforts.

Importantly, the authors found that the level of participation was both strongly and positively correlated with perceived MPA performance. Thus, villagers who were closely tied to ensuring the success of their local MPA either through attending trainings or reporting poachers, were more likely to feel their MPA was making a positive contribution.

A variety of factors were predictors of participation, including age, gender, education, food security, scientific knowledge, the degree to which the participant felt human interactions could impact the environment, how closely the participant self-identified with nature, and how much the participant personally benefited from the MPA. Men were more likely to participate than women, perhaps because men were more likely to be involved in fishing in these communities. Previous research has shown that users who self-identify with nature were more likely to engage in pro-environmental behaviors, which was confirmed by this study. Villagers with more education -- including those who could correctly identify corals as animals and mangroves as important fish nurseries -- were more like to participate. Participation was also more likely amongst those who were experiencing food and income insecurity. These factors and more (which you can read about in the full paper) were used to make a model to illustrate positive feedback loops.

As you can see in the figure on the next page, there is a strong positive relationship (0.31) between participation in an MPA planning and management process and belief in the success of that MPA. In short, better engagement leads to increased perceptions of success. You can also see there are positive feedbacks between perceived MPA performance, participation, and social-ecological factors like scientific knowledge, education, self-identification with nature, and perceived personal benefits from the MPA. Thus, the model suggests that participation can educate resource users about the environment and how the MPA provides benefits.

(figure shown on second page)
This is a summary of: Lessons from Philippines MPA Management: Social ecological interactions, participation, and MPA performance

Accessible at: https://marxiv.org/wk83f

Authors: Julia Twichell, Richard Pollnac, Patrick Christie

Added to MarXiv: April 2018

Published: Environmental Management, 2018

Suggested Citation: Positive feedback between participation and MPA performance in the Philippines. OCTO (2018). DOI: 10.17605/OSF.IO/ PH5NA

See more MarXiv summaries at https://www.marxivinfo.org/ summaries

Share your research in MarXiv and the MarXiv Team may summarize it, just like this!

Step-by-step instructions on how to share your research in MarXiv are detailed at https://www.marxivinfo.org/ submission

Across all disciplines, papers available for free with a preprint (also known as "Green OA") are cited $30 \%$ more than the world average (DOI: 10.7287/peerj. preprints.3119v1) 


\section{MarXiv Summary}

MarXiv: The free research repository for the ocean \& marine-climate sciences

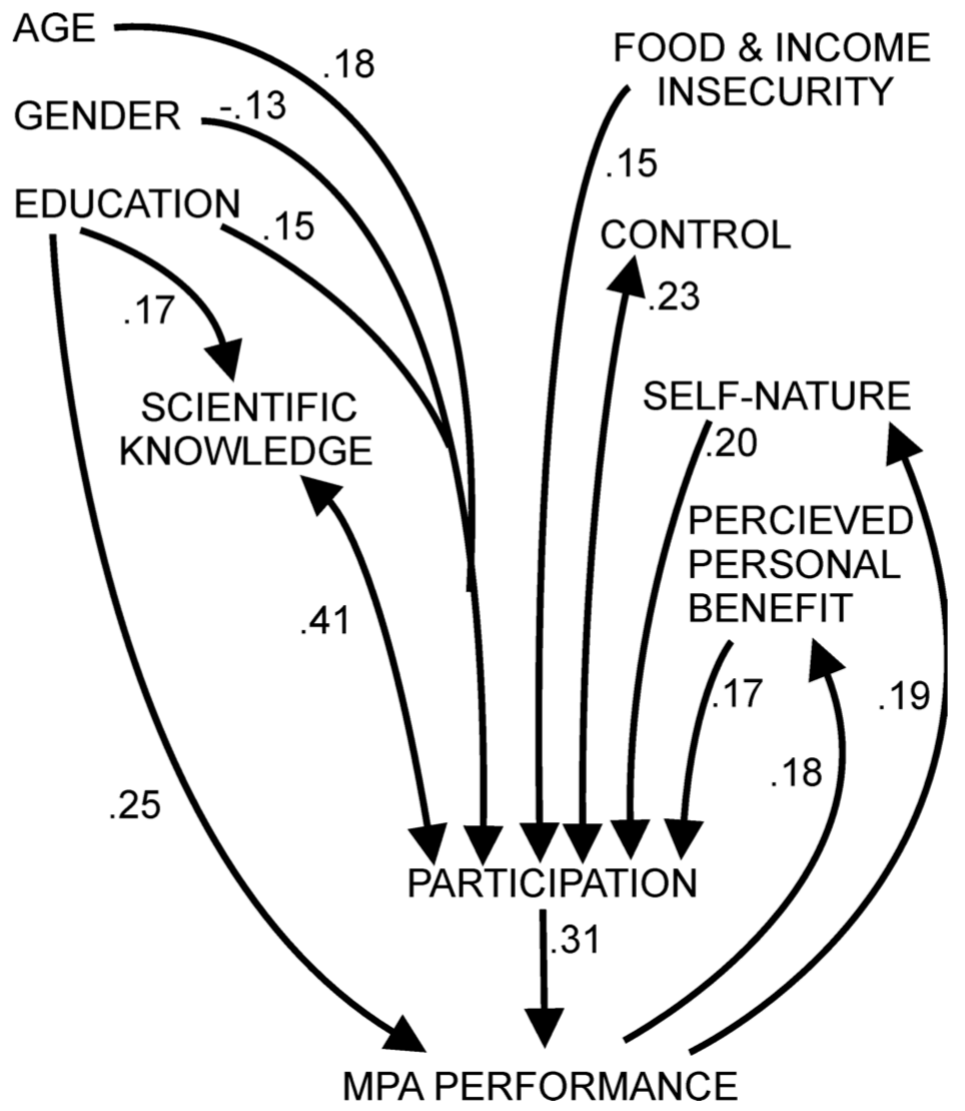

Fig. 3 Heuristic model developed from inferred relationships between variables

\section{MarXiv}

The MarXiv Team

Nick Wehner,

Director of Open Initiatives,

OCTO

Raye Evrard,

Project Manager, OCTO

Allie Brown,

Project Associate, OCTO

Sarah Carr,

Chief Knowledge Broker,

OCTO

John Davis,

President, OCTO

Correspondence

Nick Wehner

nick@octogroup.org

Financial support for MarXiv comes from the David and Lucile Packard Foundation.

The views expressed herein are those of the MarXiv Team and should not be interpreted as representing the opinions or policies of the Packard Foundation or other funders of MarXiv.

Join the MarXiv Summaries monthly newsletter at https:// oct.to/marxivsum

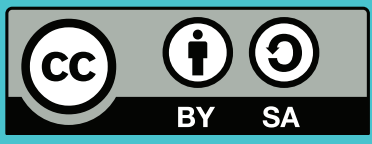

MarXiv is an ОСТO Initiative
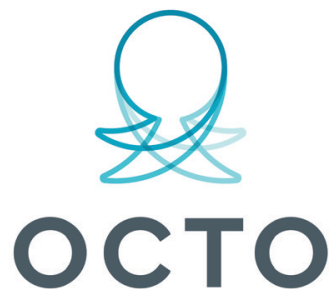

OPEN COMMUNICATIONS FOR THE OCEAN 